\section{LAS MUJERES DE ETA: ACTIVISMO Y TRANSGRESIÓN}

\author{
María Jesús Pando Canteli \\ Universidad de Deusto \\ ORCID iD: https://orcid.org/0000-0003-1272-2791 \\ mpando@deusto.es \\ María Pilar Rodríguez Pérez \\ Universidad de Deusto \\ ORCID iD: https://orcid.org/0000-0001-5905-9152 \\ maria.pilar@deusto.es
}

Cómo citar este artículo/Citation: Pando Canteli, M. J. y Rodríguez Pérez, P. (2020). Las mujeres de ETA: activismo y transgresión. Arbor, 196 (796): a554. https://doi.org/10.3989/ arbor.2020.796n2007

Recibido: 26 mayo 2018. Aceptado: 23 octubre 2019.

RESUMEN: La transgresión que supone la asociación de la violencia con las mujeres se revela en el tratamiento político y mediático que han recibido las mujeres de ETA, frecuentemente asociadas con una feminidad pervertida. Este artículo analiza la identificación del cuerpo de la mujer de ETA con la sexualidad desviada de los modelos normativos nacionalistas, explora las conflictivas relaciones de las mujeres con la violencia y se detiene en las figuras políticas y el liderazgo de Yoyes y de Idoia López Riaño. En el primer caso se analiza la transgresión representada por la presencia inasimilable de una líder de ETA tras su abandono de la actividad terrorista y tras su maternidad. En el segundo se explora su representación y la negociación política y mediática de su identidad. Si Yoyes ocupa un espacio simbólico en el imaginario político vasco, López Riaño constituye un testimonio vivo en el proceso de esta militancia femenina en ETA.

PALABRAS CLAVE: Terrorismo; transgresión; género; sexualidad; medios de comunicación.

\section{FEMALE ETA MEMBERS: ACTIVISM AND TRANSGRESSION}

Copyright: (C) 2020 CSIC. Este es un artículo de acceso abierto distribuido bajo los términos de la licencia de uso y distribución Creative Commons Reconocimiento 4.0 Internacional (CC BY 4.0).

ABSTRACT: The transgression resulting from the association of violence with women is revealed in the political and mediatic treatment that women of ETA have received, often associated with a perverted femininity. This article analyses the identification of the female ETA body with a sexuality divergent from nationalist normative models. It explores the conflictive relationship of women with violence and the political figures and leadership of Yoyes and Idoia López Riaño. In the first case, the article analyses the transgression represented by the unassimilable presence of an ETA leader after her abandonment of terrorist activity and after motherhood. In the second, it explores López Riaño's representation and the political and mediatic negotiations of her identity. If Yoyes occupies a symbolic space in the Basque political imagination, López Riaño is a living testimony in the process of this female militancy in ETA.

KEYWORDS: Terrorism; transgression; gender; sexuality; media. 


\section{PARTICIPACIÓN Y PERCEPCIÓN DE LAS MUJERES EN ORGANIZACIONES TERRORISTAS}

El terrorismo es uno de los fenómenos más complejos y más difíciles de definir por la imprecisión de sus límites en terrenos tales como el político, el social y el religioso, y especialmente por la posición ideológica de quien lo define. Sea cual sea su concreción particular, la figura del terrorista se articula como el epítome de la transgresión absoluta por la amenaza física, económica, política y moral que impone a las sociedades en las que actúa (Malvern y Koureas, 2014, p. 72). La percepción social y moral de la transgresión y de la amenaza que el terrorismo representa se acentúa cuando la figura destacada en un grupo violento es una mujer. Como explica Susan N. Herman, hay una tendencia constante a asociar inmediatamente el terrible impacto que causa el terrorismo en las mujeres en lugar de admitir su participación activa como agentes (Herman, 2009, p. 260). En los últimos años se advierten esfuerzos significativos por parte de la crítica para estudiar el papel de las mujeres como responsables de la violencia (Eager, 2008; Poloni-Standinger y Ortbals, 2013; Sjoberg y Gentry, 2007, entre otros). A pesar de que siempre ha existido una participación activa de las mujeres en diversas organizaciones terroristas, y de la atención que está recibiendo en los últimos años, por ejemplo, su actividad en movimientos yihadistas, las mujeres son frecuentemente percibidas en el imaginario social como incapaces de participar activa y voluntariamente en ataques terroristas (Agra Romero, 2012; Cruise, 2016; Nacos, 2005). Cindy Ness (2005) incide en esta misma idea y apunta a la transgresión que supone la participación de las mujeres en actos violentos terroristas, ya que supone una violación de las nociones convencionales de la normatividad de relaciones de género y de poder (p. 354). Por su parte, cabe destacar la aportación de Eulalia Pérez Sedeño (2012), quien incide en que la idea de que las mujeres solo desempeñan, en todo caso, labores de apoyo corresponde a unos estereotipos de género que asocian a las mujeres con la ternura, la compasión, la sensibilidad, el cariño, el cuidado, el afecto y la sumisión. Dichos estereotipos extienden la creencia de que las mujeres son apolíticas, que la división del trabajo entre mujeres y hombres se basa en diferencias físicas y en la distinta capacidad de unas y otros para llevar a cabo ciertos actos y en que el propósito y función primaria de una mujer es ser madre y esposa, lo que refuerza estereotipos tradicionales y no permite una visión realista de situaciones en las que las mujeres actúan violentamente, como es el caso de las terroristas islámicas, por ejemplo.
La transgresión que representa la participación de las mujeres en el terrorismo se deriva de una distinción estereotipada de lo masculino y lo femenino, y de la asociación casi exclusiva de la violencia con los hombres. Herman señala tres estereotipos sexistas que han llevado a la perpetuación de tal creencia:

First, that women are apolitical; second, that the division of labor between men and women is based on physical differences in the ability of men and women to undertake certain acts; and third, that a woman's primary purpose and function is to be a mother and a wife rather than having an individual identity of her own (Herman, 2009, p. 262).

Este monográfico se articula en torno a manifestaciones y prácticas no organizadas de crítica a las normas políticas, sociales y de género. El proyecto recoge en su totalidad planteamientos y experiencias que han quedado borrados u olvidados en los tradicionales relatos políticos, sociológicos e historiográficos sobre diversas actividades, al reproducir una imagen exclusivamente masculina del activista político. Este artículo, en particular, muestra las formas en las que la transgresión de la militancia y el activismo de mujeres en la organización terrorista ETA fue interiorizada y expuesta por parte del estamento político y de los medios de comunicación. Y lo hace centrándose en dos figuras paradigmáticas: Dolores González Catarain, Yoyes, e Idoia López Riaño, la Tigresa.

El pensamiento binario de género y la reproducción dicotómica de los estereotipos son visibles en las maneras en que se imaginan las mujeres terroristas; son percibidas como criaturas crueles y frías sin sentimientos humanos o como simples acompañantes de sus amantes masculinos, lo que supone una diferencia cualitativa en la apreciación social y política cuando es una mujer la que comete un acto violento. Laura Sjoberg y Caron Gentry describen en la siguiente cita las narrativas más comunes para caracterizar las mujeres en actividades violentas:

Women engaged in proscribed violence are often portrayed either as 'mothers', women who are fulfilling their biological destinies; as 'monsters', women who are pathologically damaged and are therefore drawn to violence; or as 'whores', women whose violence is inspired by sexual dependence and depravity (Sjoberg y Gentry, 2007, p. 12).

La excepcionalidad que su mera presencia supone en actividades violentas convierte a las mujeres terroristas en figuras paradójicas que resultan difíciles de incorporar a los discursos sobre el terrorismo. La 
doble transgresión que representan surge de su implicación en acciones terroristas y de su condición de mujeres, que se alejan de las prácticas violentas en el imaginario social construido en torno a diferencias esencialistas. Testimonios recogidos por militantes de ETA (varones y mujeres) insisten en la dificultad asociada a la militancia femenina, que contraviene estereotipos contra los cuales las propias mujeres militantes tenían que luchar (Reinares, 1998). La tendencia a relegarlas a tareas de "mantenimiento y recogida de información" (Reinares, 1998, p. 31) ponía de manifiesto la marcada jerarquía de la banda, algo a lo que se rebelan las propias militantes; ellas eran percibidas por sus propios compañeros de banda como elementos disruptores y provocadores hasta el punto de darse casos de acoso sexual bajo amenaza por los mismos compañeros de comando (Reinares, 1998, p. 27). Por tanto, los patrones de conducta, lejos de ser revolucionarios e igualitarios no hacían sino perpetuar el machismo subyacente en la propia sociedad vasca y el orden social tradicional, algo que la disciplina militar de la banda armada refuerza (Hamilton, 2007, p. 136). En este sentido Schraut (2014) señala que, a pesar de que en general la violencia política se enmarca en el deseo de transgredir normas políticas y de género, incluso aquellos que están a favor del terrorismo tienden a reproducir los estereotipos de género.

En la misma dirección ahonda Hamilton (2007) cuando afirma que las políticas de género de ETA son inextricables de su ideología nacionalista y sus raíces en la comunidad nacionalista de Euskadi (p. 134). Siguiendo esta ideología, a pesar de la creciente presencia de mujeres en ETA a finales de los años setenta y en los años ochenta, su participación continuó siendo interpretada como una extensión de sus vidas privadas y relaciones personales (Hamilton, 2007, p. 137). Los siguientes apartados de este artículo exploran cómo los discursos mediáticos construyen la anomalía de estas mujeres y los procesos de demonización y sexualización que se desarrollan como respuesta a lo que se percibe como una transgresión en diversos niveles del imaginario social y político.

\section{YOYES E IDOIA LÓPEZ RIAÑO: ANOMALÍA Y DEMONIZACIÓN}

Sue Malvern expone la doble transgresión que supone el hecho de ser una mujer terrorista y afirma que la violencia de las mujeres, porque contradice las normas de la conducta de género, es percibida como más excesiva que la de los hombres, amenaza a la masculinidad y frecuentemente se sexualiza (Malvern, 2014; Nacos, 2005, p. 435). Tal sexualización responde a la amenaza que la sociedad percibe ante un cuerpo femenino que es capaz de dar vida pero en este caso también de arrebatarla. Es en el foco del cuerpo femenino como dador y usurpador de vida donde convergen los casos de Yoyes e Idoia López Riaño, pero además ambas ilustran los estereotipos femeninos en su versión más polarizada y arquetípica: la madre frente a la mujer fatal.

Importa señalar que Yoyes e Idoia López Riaño representan un modelo femenino inasimilable y transgresor, pero mantienen diferencias significativas en sus trayectorias vitales, y ello modifica sustancialmente el modo en que son percibidas por la opinión pública. En realidad, sus experiencias no son comparables si no es porque ambas representan un momento y una identidad asociada a la lucha armada en el País Vasco. Mientras Yoyes representa un activismo centrado en los movimientos revolucionarios de los años 70, muy marcado por ideologías de izquierdas y con un compromiso político inequívoco, López Riaño representa lo más duro e incomprensible de la acción terrorista, los asesinatos colectivos de los años 80 y 90, la violencia despiadada e injustificada, la actitud cínica y despectiva frente a la justicia, el impacto mediático y la construcción de una identidad basada en este impacto. Como resultado y como proyección política, mediática y social, de Yoyes sobrevive una identidad construida como víctima y no como verdugo, marcada por su asesinato y por el hecho de no haber tenido delitos de sangre. El suyo es el mito de la maternidad condenada: violencia y maternidad son en el imaginario colectivo conceptos antagónicos; la madre no puede ser asesina. López Riaño, por su parte, se aleja del mito de la maternidad y encarna el de la femme fatale, contraria a la maternidad en su esencia como sexualidad exacerbada y estéril, y que en su radicalización encaja perfectamente con los modelos normativos asociados a la violencia terrorista. En este sentido, la representación de López Riaño es coherente en tanto en cuanto se construye una imagen de mujer anómala y agresiva, cuya hiper sexualización se ajusta también a su identidad violenta. Como se verá, la proyección de su violencia se ve asociada a lo que se percibe y documenta como una sexualidad insaciable, que la convierte en devora hombres y ejecutora, todo a la vez. A diferencia de Yoyes, López Riaño representa la no-maternidad, la feminidad sexualizada y la materialización de la violencia terrorista en el cuerpo femenino. 


\section{LA VIDA Y LA MUERTE DE YOYES: ANOMALÍA Y TRANSGRESIÓN}

Este apartado analiza la excepcionalidad de la figura de Yoyes, que encarnó la inasumible figura de una militante ETA que decidió abandonar la organización y convertirse en madre en el exilio. La figura de Yoyes transgrede la estereotipada imagen de las mujeres que han participado en organizaciones terroristas como simples acompañantes en muchos aspectos, y el más destacado es que su liderazgo político y su militancia han sido ampliamente reconocidos. Ha recibido gran atención crítica, aunque los estudios más significativos se refieren a la representación cinematográfica de la figura histórica en la película Yoyes, de Helena Taberna (2000). Elisa Costa-Villaverde (2007), Ann Davies (2009), Santiago de Pablo (2012), María Pilar Rodríguez (2002), Carlos Roldán (2011) y Rob Stone y María Pilar Rodríguez (2015), entre otros, subrayan el tono feminista empleado por Taberna al abordar el retrato fílmico de su protagonista, y el afán por reconciliar los aspectos aparentemente insolubles de la actividad política de la protagonista y su vida privada.

Yoyes nació en Ordizia (Gipuzkoa) en 1954, y desde la adolescencia mostró simpatía por la ideología de la izquierda abertzale. Compaginó sus estudios de Magisterio en San Sebastián con actividades de apoyo a ETA como militante legal, hasta que en 1973 tuvo que trasladarse a Francia. La anomalía que constituye la vida de Yoyes viene marcada, en primer lugar, por su ascenso en la cúpula de ETA hasta llegar a ser una de sus dirigentes más destacadas. En 1978 formaba parte del Comité Ejecutivo de la rama militar de ETA. Cuando en diciembre de 1978 su compañero y mentor José Miguel Beñaran, Argala, fue asesinado a manos del BataIlón Vasco Español, Yoyes pasó a ocupar su puesto al frente de la dirección política de ETA-militar. Tras un distanciamiento progresivo de la llamada línea dura de ETA, decidió abandonar la organización y exiliarse en México. En agosto de 1985, al no haber ninguna causa judicial abierta contra ella y de acuerdo con la Ley de Amnistía de 1977 decidió regresar a Euskadi, con su marido y su hijo pequeño (Rodríguez, 2016). Yoyes fue asesinada por ETA el 10 de septiembre de 1986 en su localidad natal en presencia de su hijo de tres años.

Es precisamente el estatus excepcional de Yoyes como miembro del comité ejecutivo de ETA y sus decisiones a nivel político las razones motivaron la amplificación y la resonancia de sus movimientos, que eventualmente fueron instrumentales en el proceso que desencadenó su muerte. Contrariamente a la percepción tradicional de la participación de las muje- res en organizaciones violentas, las acciones de Yoyes nunca fueron vistas como tangenciales al centro de la política. Su conciencia política y su excepcionalidad como figura femenina estaban presentes desde el principio; Koldo Iztueta relata la integración de Yoyes en uno de los taldes (grupos o comandos) durante el curso académico 1971-1972, cuando tenía 17 años. El talde estaba formado por solo mujeres e Iztueta destacaba el papel prominente de Yoyes y su convicción ideológica, que era muy fuerte (Garmendia Lasa, González Katarain, González Katarain, Garmendia Lasa y Dorronsoro, 2009, p. 62). La vida y la muerte de Yoyes reflejan la imposibilidad de responder a la pregunta que Rob Stone y Helen Jones plantean: "Where to place the female gudari?' (2004, p. 48). En efecto, si el lugar de la gudari o luchadora en la terminología de la izquierda abertzale supone un no-lugar precisamente por la trasgresión que representan las mujeres cuando están a cargo de la acción, en el caso de Yoyes su existencia se vio marcada por la imposición de un constante desplazamiento y de un proceso de desterritorialización permanente. A sus exilios en México y en Francia hay que añadir la imposibilidad del regreso a su tierra natal, que se vio marcada por la amenaza desde el comienzo. El asesinato de Yoyes se debió a dos razones principales y ambas dan fe de la imposibilidad que para el mundo de la izquierda radical supuso la anomalía que representaba. La primera fue su maternidad, que suponía una ruptura con las políticas de género normativas del nacionalismo vasco, y la segunda fue la dimensión pública que adquirió su regreso, a pesar de sus denodados esfuerzos por ocultarse en el anonimato y por fomentar su propia invisibilidad.

Begoña Aretxaga afirma que la decisión de Yoyes de dejar el activismo terrorista para ocultarse en el anonimato de una vida civil, y especialmente su decisión de ser madre constituyó para los líderes de ETA un choque amenazante de identidades de género:

Yoyes was an anomaly in the radical nationalist world [...]. Yoyes was treated as a hero and as a traitor, but she was a mother at the same time. A mother, by definition, cannot be a hero or a traitor in the cultural context of radical nationalism; she is beyond these categories. Yoyes collapsed gender differentiations at a moment when $\mathrm{ETA}(\mathrm{M})$ needed them more rigidly than ever (Aretxaga y Zulaika, 2005, p. 161).

Los diarios escritos por Yoyes y editados y publicados por sus familiares bajo el título Yoyes desde su ventana reflejan la conciencia clara que la joven sentía de la escisión entre su actividad terrorista y su condición de madre. Desde muy temprano registra en sus 
diarios la convicción de que solo podría convertirse en madre en una segunda etapa después de su abandono de la organización: "Cuando sentí que no me involucrarían de nuevo tuve a Akaitz, un niño maravilloso que me absorbió un montón" (Garmendia Lasa et al, 2009, p. 243). Yoyes percibió el embarazo y el nacimiento de su hijo Akaitz como el punto final para marcar su disociación con toda participación política $y$, como escribe en sus diarios, como una nueva vida que cerró la puerta a la muerte (Garmendia Lasa et al, 2009, p. 112). Sin embargo, lo que para Yoyes era una división clara entre el antes y el después de su pertenencia a ETA no era más que una línea muy difusa e imperceptible para sus antiguos compañeros. La relevancia que Yoyes había alcanzado dentro y fuera de ETA la hizo muy consciente de la necesidad de permanecer en silencio, de desaparecer física y políticamente para comenzar una nueva vida. ETA también estaba muy interesada en mantener el silencio acerca de su abandono; como explica Aretxaga: "From ETA's point of view, since Yoyes was a well-known, almost mythical figure, her desertion, if publicly broadcast, would be demoralizing for ETA $(\mathrm{m})$ ' sympathizers" (Aretxaga y Zulaika, 2005, p. 150).

Los medios de comunicación desempeñaron un papel destacado tras su regreso a Euskadi. En enero de 1986, uno de los principales periódicos españoles, Cambio 16, publicó un número con este titular en la portada: El regreso de la etarra. Fue precisamente la insistencia en su liderazgo y en su estatura política lo que inmediatamente provocó la percepción de ella como una traidora por parte de los nacionalistas radicales. Como explica Fernando Reinares, este modus operandi es favorecido por las organizaciones terroristas para salvaguardar su propia supervivencia:

Con frecuencia, las organizaciones clandestinas hacen esfuerzos para establecer mecanismos de sanción social e incluso de coerción física para prevenir signos de desacuerdo de quienes los apoyan (Reinares, 1998, p. 88).

Seis años después del abandono de toda actividad política, la sombra de su envergadura como líder de ETA era todavía considerable dentro y fuera de la organización. La respuesta dada por un representante de Herri Batasuna en abril de 1987 es ilustrativa de tal percepción de Yoyes como líder política y de la necesidad de la organización de mantener un sentido de obediencia disciplinaria: "Un ejército no puede permitirse el lujo de la traición de sus militantes y mucho menos de uno de sus generales" (citado en Letamendía Belzunce, 1994, p. 112).
Si la presencia de Yoyes resultó imposible de asumir, su muerte está marcada en muchos aspectos por signos de la excepcionalidad que su vida representó. La mayoría de los militantes de ETA muertos en circunstancias relacionadas con su pertenencia a la organización y la mayoría de las víctimas de ETA han sido hombres. En este contexto, hay una preeminencia de madres y viudas que ahora son instrumentales en varias asociaciones de víctimas. Sin embargo, Yoyes no era la madre presente para llorar la pérdida de su hijo; fue su propio funeral el que tuvo lugar en Oridizia. Aretxaga analiza cuidadosamente el homenaje funerario de Yoyes, que tuvo lugar un mes después de su asesinato, en el que las paradojas que marcaron su vida continuaron presentes después de su muerte. Si, como explica Joseba Zulaika, en los homenajes funerarios que se realizan ritualmente para los miembros de ETA tras el regreso del cuerpo a sus pueblos nativos, las madres tienen un papel central a menudo sosteniendo las cenizas del hijo muerto (Zulaika, 2010, p. 111), aquí se mostraba una inversión radical, ya que Yoyes era el único miembro femenino de la organización que había sido asesinado por sus antiguos compañeros. Sin embargo, como informa Aretxaga, el homenaje funerario de Yoyes se realizó de manera similar a los organizados para miembros de ETA asesinados por las fuerzas del Estado español, e incluyó la presencia de bertsolariak (improvisadores de versos) y el homenaje en la plaza principal del pueblo (Aretxaga y Zulaika, 2005, p. 157). Por todo ello, Yoyes se resiste a la categorización en términos de pertenencia a las dos partes claras y diferenciadas en el conflicto de la violencia vasca entre terroristas y víctimas. Ella fue miembro de una organización terrorista y ella fue una víctima de ETA, y ambas declaraciones son susceptibles de ser rechazadas por sectores de la población vasca. Yoyes niega la primera caracterización en un pasaje de sus diarios en el que rechaza el modo de pensar binario y dicotómico que la define como terrorista y como traidora:

No me consideré héroe, no puedo considerarme antihéroe, tampoco fui terrorista sino militante política, el hecho de no serlo no me convierte automáticamente en parte potenciante del sistema (Garmendia Lasa et al, 2009, p. 244).

Similarmente, es fácil entender que haya víctimas de ETA que no consideren que el estatus de Yoyes deba ser el mismo que el de las viudas, hijas e hijos de la violencia terrorista por su pertenencia anterior a la organización. 


\section{IDOIA LÓPEZ RIAÑO: HIPER SEXUALIZACIÓN DE LA VIOLENCIA}

No hay ninguna otra figura femenina en ETA que haya recibido más atención mediática que Idoia López Riaño. En una noticia en Deia con motivo de su excarcelación se la califica como "el icono femenino de la banda" (Deia, 11 de junio de 2017). Ha sido, sin duda, quien más ríos de tinta ha generado, no solo en la prensa diaria sino también en reportajes y en publicaciones que, como la de Antolín (2002), contribuyeron a fijar esa imagen de mujer fatal. Su representación mediática se caracteriza por los grandes titulares e incide de forma recurrente en aspectos que van más allá del hecho informativo. A diferencia del tratamiento que reciben otros miembros de ETA, varones y mujeres, a quienes se les apela con nombre completo y alias, tal y como se les identifica dentro de la banda, en el caso de López Riaño no se utiliza el alias en la banda, Margarita, sino el supuestamente dado por la policía, la Tigresa, que ha calado profundo en los medios que lo han popularizado. Es este un hecho diferenciador de no menor importancia, ya que las connotaciones del apodo incorporan un juicio, unos rasgos de carácter, un comportamiento, que estarían ausentes en, por ejemplo, el alias de Carmen Guisasola, Lourdes, o de Belén González Peñalba, Carmen, por poner solo dos ejemplos de mujeres militantes en ETA.

López Riaño genera fascinación en los medios y se ha creado una leyenda en torno a ella que más se asemeja a un guion de thriller que a una crónica política. Los hechos noticiables en torno a su persona (detención y extradición, arrepentimiento y acogida a la vía Nanclares y, finalmente, excarcelación) están relatados de manera que difícilmente se mantienen en el nivel de lo meramente informativo: "La Tigresa vuelve a casa: los miedos de una asesina múltiple al salir de la cárcel" (El Español, 13-06-2017), "Idoia, de Tigresa a gatita" (EI Mundo, 30-07-2010), "No tiene valor para mirarme a los ojos" ( $A B C$, 31-07-2010), "El sangriento historial de López Riaño" (El Correo, 13-06-2017), "Viaje al interior de la Tigresa" (El Correo, 24-07-2014) son algunos de los titulares recogidos en los últimos años.

Lejos de los estereotipos de la figura andrógina de militancia armada, sin concesiones a la frivolidad ni a la feminidad explícita y que responde a un modelo de conducta asimilado al de "la compañera de...", López Riaño es representada como una mujer coqueta y de sexualidad exacerbada, ajena a los modelos de feminidad del espacio simbólico vasco, donde la madre sigue gozando de un importante peso simbólico; madre que, junto a la compañera, apuntalan este universo abertzale (Hamilton, 2000). El caso de López Riaño se hace patente en este contexto, en el que se presenta como un elemento disruptivo y transgresor, no solo para las fuerzas y cuerpos de seguridad del Estado sino para la propia banda, cuyos miembros están acostumbrados a la militante subalterna, con tareas de supervisión de infraestructuras y no a la militante de primera fila dispuesta a apretar el gatillo. Su actitud representa una amenaza porque desestabiliza el orden social impuesto en la banda, el cual refuerza el rol de mujeres compañeras y obedientes, sin ambición de usurpar las responsabilidades y obligaciones que por derecho natural los varones tienen asignadas (Hamilton, 2007, pp. 133-139).

El discurso con frecuencia asocia su historial delictivo a su condición de mujer, a su atractivo físico y a su sangre fría para matar. Es así como se ha ido construyendo este mito de femme fatal: amenazadora, terrible y fascinante a la vez para quienes construyen estas crónicas. Pocos periodistas pueden presumir más que Matías Antolín de hacer el relato más sensacionalista y machista de la militante de ETA. En él confluyen todos los tópicos asociados a la mujer fatal, en una suerte de tono efectista que lo aleja del periodismo riguroso y lo hermana con el amarillismo más populachero. A López Riaño Antolín la presenta bajo el subtítulo de "la cama y la pistola", como una mujer que:

[...] siempre me produjo miedo y fascinación. Es indomable como una orquídea de acero inoxidable [...] se infiltraba como una carcoma entre los "maderos". Se movía entre los hombres como una pantera, se escurría sigilosa entre los policías como una serpiente venenosa, se enredaba como una araña de ojos azules (Antolín, 2002, p. 19).

En su acercamiento no académico a las mujeres en ETA, el libro de Antolín exhibe un amplio catálogo de comentarios sexistas en su caracterización de las mujeres miembros de ETA. Colectivamente, las mujeres del Comando Madrid (el grupo más activo y letal en ETA durante la década de 1980) son así retratadas:

No tenían mucha capacidad de decisión y casi todas fueron un nudo conflictivo en la convivencia. La mayoría (Belén, Idoia, Ainhoa, Soledad...) eran arrogantes, dominantes, manipuladoras, egoístas, mentirosas, superficiales, carentes de remordimientos, sin escrúpulos y, sobre todo, asesinas (Antolín, 2002, p. 139).

Para proporcionar una ilustración individual dentro de ese conjunto cuya descripción incluye los estereotipos peyorativos asociados tradicionalmente a las mujeres, Idoia López Riaño es descrita por Antolín 
como una mujer sensual, una "esclava de su cuerpo y su cabello" (Antolín, 2002, p. 20) y, al explicar más la actividad de López Riaño, explícitamente rechaza su compromiso político o inclinación ideológica, y declara abiertamente:

No tenía ni idea de política, se hizo de ETA como pudo acabar de asaltadora de bancos. Es altiva, desafiante y coqueta. Pertenece a la estirpe de ese tipo de mujeres que sueñan con trenes llenos de soldados. (Antolín, 2002, p. 30).

Las mujeres terroristas a menudo son vistas como apolíticas y carentes de ideología; curiosamente las mujeres que MacDonald entrevistó en el País Vasco a fines de la década de 1980, cuyos testimonios están incluidos en el primer capítulo del libro titulado Entre las mujeres de ETA, rechazan firmemente la idea de que se involucraron en la organización a través de novios y amantes (MacDonald, 1991, p. 11).

Uno de los episodios que más ha calado en el imaginario colectivo y que ha contribuido a crear la leyenda y la imagen perversa de López Riaño es aquel que relata la obsesión que la etarra tenía por seducir a los policías: "Se dice -prosigue Antolín- de la Tigresa que cuando les tenía debajo su mayor deseo era "pegar un tiro en la boca a esos cabrones»" (Antolín, 2002, p. 20). Es cierto que esta fantasía, cuyo relato aparece en varias publicaciones, se presenta más como una expresión de la masculinidad amenazada que como una realidad. ¿Acaso Idoia López Riaño asesinó realmente a alguna de sus conquistas mientras las tenía entre sus piernas? Curiosamente esta ensoñación ha iconizado a la Tigresa. Curiosamente también nadie ha podido confirmar la autenticidad de la cita. La expresión "se dice" o "la leyenda..." (Antolín, 2002, p. 20) pone de manifiesto que la anécdota se ha ido construyendo sobre la verosimilitud de un estereotipo: "Su exultante belleza y su sangre fría convirtieron a la Tigresa en uno de los personajes más temidos de ETA" (Antolín, 2002, p. 21, cursiva nuestra). Interesa resaltar el término personaje puesto que realmente el discurso periodístico no hace sino construir una identidad, un personaje de ficción donde confluyen miedos y ansiedades en torno a esta feminidad desviada que transgrede espacios de domesticidad y normas de conducta; difícilmente se puede abordar la figura de López Riaño en la prensa sin asumir este punto de partida de personaje construido a priori por una mirada masculina y sobre el que se van añadiendo descripciones, escenas y crónicas que refuerzan el mito, la construcción de una identidad mediática.
Como figura transgresora López Riaño opera a varios niveles: por una parte, la feminidad marcada, atractiva y autónoma, frente al modelo de madre y compañera, más mujer que las demás mujeres en su atractivo sexual y no asimilable al tipo común de compañera de. Por otra parte, la maketa: de origen español, padres salmantinos y extremeños, primera generación, más vasca que los vascos, extrema identidad nacionalista. Muestra una identidad individual marcada y transgresora en estos tres extremos: étnico (no pertenece a la raza), sexual (contraviene los estereotipos de mujer nacionalista neutra sexualmente hablando y de aspecto físico común) y de género (se muestra supuestamente más violenta que sus compañeros varones) utilizando, de acuerdo al relato mediático, sus armas de mujer como armas letales. Es sobre estas desviaciones que confluyen en lo que Rodríguez Lara denomina "hibridación de género-conflicto" (2013, p. 152) sobre las que se construye su identidad transgresora. Sobre este entramado los medios han logrado elaborar una imagen impactante, difícilmente cuestionable por debajo de todos los clichés que han ido andamiando a la figura asesina y letal, anómala y perturbadora que es la Tigresa.

En este relato exacerbado confluyen los miedos y las ansiedades no solo de cuerpos policiales sino también de los varones de sus propias filas. Se sabe que Soares Gamboa, antiguo compañero de comando, contribuyó notablemente a hiperbolizar la imagen rebelde, indisciplinada y letal de López Riaño en el libro Soares Gamboa. Agur, ETA. El adiós a las armas de un militante histórico (1997), publicado también por Matías Antolín. Aquí se recogen numerosos testimonios de la convivencia en el comando Madrid con López Riaño que han nutrido varias de las crónicas más impactantes de los últimos años. El periódico El Confidencial, en su edición del 13 de junio de 2017, con motivo de la inminente excarcelación de López Riaño tras haber cumplido condena, publica un artículo bajo el titular "Era esclava de su cuerpo y de su cabello": el etarra Soares Gamboa habla de la Tigresa. En el artículo se la retrata como indisciplinada, histérica, informal, inconstante, coqueta, impetuosa, manipuladora y ambiciosa: "no he salido de Euskadi para matar guardias civiles, estoy en Madrid para matar generales" dice Antolín que dijo Gamboa que había dicho López Riaño (Alonso, 2017, 13 de junio). Si bien el hecho de que el artículo reproduzca todos y cada uno de los estereotipos más trillados de la misoginia clásica ya sería objeto de análisis en sí mismo, lo que llama la atención aún más es que la etarra debería ser noticia por su excarcelación inminente por cumplimiento de condena y no 
por lo que un ex compañero opine de su indumentaria, aspecto físico o carácter. La desviación de atención del hecho judicial a la crónica amarilla, con especial énfasis en la anomalía del personaje, pone de manifiesto la distorsión y manipulación que los medios hacen de la información e ilustra la construcción sesgada de identidades de género, en torno a rasgos estereotipados que nunca hubieran tenido relevancia si el excarcelado hubiera sido un varón. Crónicas semejantes se publicaron en varios medios esos días previos y, dos días más tarde, López Riaño abandonaba la cárcel frente a una opinión pública hostil y una expectación mediática sin precedentes en los casos de sus compañeros varones. Todo ello no tanto por la dimensión tremenda de sus asesinatos y las incertidumbres en torno a su reinserción, cuanto por el personaje que de la Tigresa se había construido durante años. En el artículo ya mencionado anteriormente, "Idoia, de Tigresa a gatita", se cuestiona el arrepentimiento de la terrorista:

Sanguinaria, fría, calculadora, una 'tigresa' [...] devoradora de hombres en sus buenos tiempos, dicen [...] sus ojos verdes y su pelo ensortijado, armas de mujer con las que salía a quemar la noche aquí y allí (El Mundo, 29-07-2010).

En esta misma línea Txetxu Aguado (2017) va un paso más allá al afirmar que "la lucha antiterrorista identificó el cuerpo de la mujer de ETA con la sexualidad desviada de la norma para las mujeres" (p. 154). Aguado identifica la masculinidad con el aparato del Estado, de forma que la mujer etarra ponía de manifiesto una masculinidad amenazada por una feminidad desviada. Si bien el cuerpo de la mujer etarra supone un espacio de conflicto en la lucha antiterrorista, no es menos cierto que este uso del cuerpo femenino es común a todo conflicto. Hubo otros y otras tanto o más sanguinarios, cínicos o fanáticos, pero a nadie como a ella se le enjuicia y castiga por mostrar unos patrones de conducta contrarios a los estereotipos de género en la militancia armada.

Este relato prácticamente homogéneo de los medios contrasta frontalmente con el escasísimo material testimonial de la propia López Riaño. Apenas saltó a la prensa la carta que escribió al juez Grande Marlaska en 2015 y solo se hizo público algún fragmento en el que la etarra hacía valer su buena conducta, mostraba su arrepentimiento y ponía en valor las numerosas actividades carcelarias en las que se había implicado desde entonces. El Diario Vasco en su edición del 15 de noviembre de 2015 recoge parte de este texto, en el que hace un recorrido exculpatorio por su trayectoria:
Me metí en ETA muy joven, llena de ideas románticas e idealistas y los que me captaron supieron enseguida cómo hacerme elegir: 'Con nosotros puedes salvar a un pueblo'. Así de estúpidamente me dejé llevar, y así de dolorosamente sigo sintiéndolo en cada poro de mi piel y en cada latido de mi corazón. El inmenso, terrible y doloroso error que cometí por falta de madurez, por creer de verdad que se me necesitaba más como miembro de ETA. Eso es algo que no me dejará nunca, esa inmensa decepción conmigo misma, ese irreparable error lo siento cada vez que respiro (Balín, 2015, 16 de noviembre).

Los extractos que recoge el Diario Vasco muestran a un sujeto que parece aceptar responsabilidades y reivindica un trato igualitario respecto a otros presos de ETA arrepentidos. Ante la repetida negativa de concederle beneficios penitenciarios por la gravedad de los hechos delictivos y su aparente falta de arrepentimiento, López Riaño denuncia los "informes falseados por el subdirector de tratamiento" y se lamenta de que su caso tenga un trato diferente precisamente como consecuencia del impacto mediático que genera, pidiéndoles a los jueces que aparquen "prejuicios hacia mi persona" y "busquen la equidad y verdad" para concluir con una cita: "Maquiavelo declaraba con razón que todos ven lo que aparento, pocos ven lo que soy".

¿Y quién es Idoia López Riaño, Tigresa, Margarita o últimamente solo Idoia? Independientemente del discurso mediático que ha construido al personaje de la Tigresa, poco se sabe del sujeto que hay detrás de este. En un artículo publicado en La Razón el día anterior a su puesta de libertad en 2017, el titular informa con un tono muy crítico de que "La Tigresa teoriza sobre la violencia de género tras matar a 23 personas", para añadir en el subtítulo y con una foto de media página: "Mañana la etarra abandona la cárcel tras cumplir 23 años de condena, uno por cada víctima. Ha estudiado valenciano, Tai Chi, y escrito un prólogo sobre casos de maltrato" (Zuloaga, 2017, 12 de junio).

El prólogo al que se refiere la noticia es el de una colección de relatos, En el umbral del dolor, en el que López Riaño traza un semblante de sí misma como persona con inquietudes y una sensibilidad muy desarrollada hacia el dolor ajeno, encarnado en todas aquellas mujeres de las que habla, compañeras carcelarias y que han sufrido en sus vidas la violencia, el dolor y el abandono. Sin duda este semblante de Idoia López Riaño produce cierta perplejidad y choca frontalmente con el de la Tigresa, dando una vuelta de tuerca más al carácter transgresor del que se habla al comienzo de 
este artículo y a la construcción polarizada de identidades en conflicto. De asesina inmisericorde y fanática a defensora de los derechos de las mujeres a través de una serie de relatos biográficos que ilustran su capacidad para la narrativa y para penetrar en el sufrimiento ajeno, erigiéndose en voz de las sin voz. Reivindica, en un tono autobiográfico, hablar de "las debilidades humanas, de los sentimientos, de lo que puede llegar a suponer para una mujer vivir encasillada, impotente, tolerando todo lo que una vida estereotipada nos marca a cada una" (López Riaño, 2012). El abandono de la disciplina de la banda y su posterior expulsión de ETA pudieron facilitarle no solo la relación más estrecha con otras reclusas comunes y un espacio de comunión en el que sus "propias experiencias se unen a las de las demás para crear un todo en el que nos confundimos, ya sin identidades, sólo con un factor común: el dolor", sino todo un proceso de reconstrucción identitaria. Las experiencias pasadas adquieren una nueva dimensión en esos nuevos espacios de encuentro y negociación, en los que se reclama

[...] una perspectiva interior de las propias mujeres, algo que nos toca tener en cuenta a nosotras mismas, cuando seamos capaces de hacerlo. Aún debemos quitamos muchas máscaras, asumir demasiadas actitudes erróneas y enfrentarnos a ellas, cambiarlas porque se han convertido en hábitos y costumbres que nos llevan a callejones sin salida en nuestras relaciones con los hombres, con todo el mundo en general. Y también con nosotras mismas. Necesitamos una toma de conciencia. Y será doloroso, pero saludable y liberador. Algunas de nosotras ya estamos pasando por ello (Prólogo de El umbral del dolor, 2012).

El texto habla de procesos de reconstrucción emocional a través de la narrativa y del carácter liberador y purificador de la palabra. Las mujeres cuyos testimonios constituyen los relatos del libro son a la vez víctimas y perpetradoras de violencia, asesinas muchas de ellas que han experimentado los límites del desamor, la crueldad y el abandono, cómo la violencia engendra violencia y cómo solo desde la construcción de su propio discurso, desde su autonomía y no la he- teronomía a la que han estado sometidas, se puede llegar a un proceso transformador y reparador.

\section{CONCLUSIÓN}

La transgresión que representan estas dos mujeres se ve claramente articulada en la proyección política, mediática y social que han recibido. Dentro del País Vasco ninguna de las dos se adecúa al prototipo de género propio de la ideología tradicional nacionalista, y ni siquiera a los modelos y prototipos que perpetuó la llamada izquierda abertzale y que no distaban considerablemente de esa imagen arquetípica de las mujeres como madres o como apoyo del gudari, pero alejadas por lo común de la acción y del liderazgo. Fuera del País Vasco, la prensa y las publicaciones acentúan el sensacionalismo ligado a su regreso (Yoyes) y la hiper sexualización y la condena (López Riaño). Al alejarse de los modelos normativos de la feminidad establecida y al confundir los compartimentos estancos en los que -consciente o inconscientemente- se alojan las únicas identidades posibles para las mujeres, ambas se muestran como sujetos que acusan las tensiones propias de la transgresión. Las dos vulneran las normas de la sexualidad y la feminidad normativa al abrazar un espacio de violencia y de actividad política radical; se convierten en identidades complejas donde confluyen los miedos y las ansiedades tanto de un universo abertzale atrapado en sus propios prejuicios y recelos, como de una sociedad civil indignada y perpleja. Durante décadas, el terrorismo en el País Vasco se identificó con unas prácticas y unos modelos de comportamiento exclusivamente masculinos, e incluso el relato literario y fílmico raramente representó a las mujeres y ello acentuó el carácter transgresor que figuras como las estudiadas en este artículo representaron.

\section{AGRADECIMIENTOS}

Este trabajo se ha realizado en el marco del proyecto Género, compromiso y transgresión en España, 1890-2016 (FEM2016-76675-P), financiado por el Ministerio de Economía y Competitividad. 


\section{BIBLIOGRAFÍA}

Agra Romero, M. X. (2012) Con armas como armas: la violencia de las mujeres. Isegoría, 46, pp. 49-74.

Aguado, T. (2017). Sexualidades desviadas: la representación de la mujer terrorista. En: Rodríguez, M. P. (ed.) Mujeres víctimas del dolor y la violencia terrorista. Madrid: Biblioteca Nueva, pp. 153-170.

Alonso, J. M. (2017, 13 de junio). Era esclava de su cuerpo y su cabello: el etarra Soares Gamboa habla de la Tigresa. El Confidencial [En línea]. Disponible en https://www.elconfidencial.com/espana/2017-06-13/retrato-eta-idoia-lopeztigresa-comando-madrid-esclava-cuerpo-cabello_1396321/

Antolín, M. (2002). Mujeres de ETA. Piel de Serpiente. Madrid: Temas de Hoy.

Antolín, M. (1997) Soares Gamboa. Agur, ETA. El adiós a las armas de un militante histórico. Madrid: Temas de Hoy.

Aretxaga, B. y Zulaika, J. (2005). States of Terror: Begoña Aretxaga's essays. Reno, Nevada: Center for Basque Studies, University of Nevada.

Balín, M. (2015, 16 de noviembre). 'La Tigresa': «El inmenso e irreparable error de entrar en ETA lo siento cada vez que respiro». El Diario Vasco. [En línea]. Disponible en https://www. diariovasco.com/politica/201511/16/ tigresa-inmenso-irreparable-error20151116112625-rc.html

Costa-Villaverde, E. (2007). Yoyes and Extranjeras, by Helena Taberna, stories of women, displacement and belonging. Studies in European Cinema, 4 (2), pp. 85-97.

Cruise, R. S. (2016). Enough with the Stereotypes: Representations of Women in Terrorist Organizations. Social Science Quarterly, 97 (1), pp 33-43.

Davies, A. (2009). Woman and home: gender and the theorization of Basque (national) cinema. Journal of Spanish Cultural Studies, 10 (3), pp. 359-72

Eager, P. (2008). From Freedom Fighters to Terrorists: Women and Political Violence. Cornwall: Ashgate.

Garmendia Lasa, E., González Katarain, G., González Katarain, A., Garmendia Lasa, J. y Dorronsoro, J. (2009). Yoyes desde su ventana. Irun: Alberdania.
Hamilton, C. (2000). Re-membering the Basque nationalist family: daughters, fathers and the reproduction of the radical nationalist community. Journal of Spanish Cultural Studies, 1 (2), pp 153-171.

Hamilton, C. (2007). Women Armed Activists in ETA. Feminist Review, 87, pp. 132-148.

Herman, S. N. (2009). Women and Terrorism. Keynote Address. Women's Rights Law Reporter, 31, pp. 258-267.

Idoia, de "Tigresa" a "Gatita" (2010, 29 de julio). El Mundo [En línea]. Disponible en http://www.elmundo.es/elmundo/2010/07/29/espana/1280433425. html

Letamendia Belzunce, F. (1994). Historia del nacionalismo vasco (vol. 3. ETA y el gobierno del PSOE (1982-1992)). San Sebastián: R\&B.

López Riaño, I. (2012) El umbral del dolor. [En línea]. Disponible en: https://boletintokata.wordpress.com/2012/06/05/ idoia-lopez-riano-el-umbral-del-dolornovedad-editorial/

MacDonald, E. (1991). Shoot the Women First. New York: Random House.

Malvern, S. (2014). Femininity, feminism and the terrorist. En: Malvern, S. y Koureas, G. (eds.), Terrorist transgressions: gender and the visual culture of the terrorist. London: I. B. Tauris, pp. 39-55.

Malvern, S. y Koureas, G. (2014). Terrorist Transgressions: Exploring the Gendered Representations of the Terrorist. Historical Social Research, 39 (3), pp. 67-81.

Nacos, B. L., (2005). The Portrayal of Female Terrorists in the Media: Similar Framing Patterns in the News Coverage of Women in Politics and in Terrorism. Studies in Conflict and Terrorism, 28, pp. 435-451.

Ness. C. (2005). In the Name of the Cause: Women's Work in Secular and Religious Terrorism. Studies in Conflict \& Terrorism, 28, pp. 353-373.

Pablo, S. (2012). The Basque nation onscreen: cinema, nationalism, and political violence. Reno, Nevada: Center for Basque Studies, University of Nevada.

Pérez Sedeño, E. (2012). Terrorismo y es tereotipos de género. Isegoría, 46, pp. 233-247.
Poloni-Standinger, L. y Ortbals, C. (2013). Terrorism and Violent Conflict. Women's Agency, Leadership, and Responses. New York: Springer.

Reinares, F. (1998). Terrorismo y antiterrorismo. Barcelona: Paidós Ibérica.

Rodríguez, M. P. (2002). Female Visions of Basque Terrorism: Ander eta Yul by Ana Díez and Yoyes by Helena Taberna. En: Ferrán, O. y Glenn, K. (eds.), Women's narrative and film in twentieth-century Spain: a world of difference(s). New York: Routledge, pp. 155-167.

Rodríguez, M. P. (2016). From dispossession to reparation: Yoyes thirty years after her death. Revista Internacional de Pensamiento Político, 11, pp. 369-381.

Rodríguez Lara, Z. (2013). Las mujeres de ETA en la prensa escrita. Mediatika, 14, pp. 151-167.

Roldán Larreta, C. (2011). Yoyes: historia y vicisitudes de un proyecto cinematográfico. Sancho el Sabio. Revista de cultura e Investigación vasca, 34, pp. 135-156.

Schraut, S. (2014). Gender and the terrorist in historiography. En: Malvern, S. y Koureas, G. (eds.), Terrorist transgressions: gender and the visual culture of the terrorist. London: I. B. Tauris, pp. 57-71.

Sjoberg, L. y Gentry, C. E. (2007). Mothers, Monsters, Whores: Women's Violence in Global Politics. London: Zed Books.

Stone, R. y Jones, H. (2004). Mapping the gendered space of the Basque Country. Studies in European Cinema, 1 (1), pp. 143-155.

Stone, R. y Rodríguez, M. P. (2015). Basque Cinema: A Cultural and Political History. London: I. B. Tauris.

Zulaika, J. (2010). Terrorism. The Self-FulfiIling Prophecy. Chicago: The University of Chicago Press.

Zuloaga, J. M. (2017, 12 de junio). «La Tigresa» teoriza sobre violencia de género tras matar a 23 personas. La Razón. [En línea]. Disponible en http://www. larazon.es/espana/la-tigresa-teorizasobre-violencia-de-genero-tras-matara-23-personas-OB15361395 